\title{
Severe Toxicity in Nonhuman Primates and Piglets Following High-Dose Intravenous Administration of an Adeno-Associated Virus Vector Expressing Human SMN
}

\author{
Christian Hinderer, Nathan Katz, Elizabeth L. Buza, Cecilia Dyer, Tamara Goode, \\ Peter Bell, Laura K. Richman, and James M. Wilson* \\ Gene Therapy Program, Department of Medicine, University of Pennsylvania Perelman School of Medicine, Philadelphia, Pennsylvania.
}

Neurotropic adeno-associated virus (AAV) serotypes such as AAV9 have been demonstrated to transduce spinal alpha motor neurons when administered intravenously (i.v.) at high doses. This observation led to the recent successful application of i.v. AAV9 delivery to treat infants with spinal muscular atrophy, an inherited deficiency of the survival of motor neuron (SMN) protein characterized by selective death of lower motor neurons. To evaluate the efficiency of motor neuron transduction with an AAV9 variant (AAVhu68) using this approach, three juvenile nonhuman primates (NHPs; aged 14 months) and three piglets (aged 7-30 days) were treated with an i.v. injection of an AAVhu68 vector carrying a human $S M N$ transgene at a dose similar to that employed in the spinal muscular atrophy clinical trial. Administration of $2 \times 10^{14}$ genome copies per kilogram of body weight resulted in widespread transduction of spinal motor neurons in both species. However, severe toxicity occurred in both NHPs and piglets. All three NHPs exhibited marked transaminase elevations. In two NHPs, the transaminase elevations resolved without clinical sequelae, while one NHP developed acute liver failure and shock and was euthanized 4 days after vector injection. Degeneration of dorsal root ganglia sensory neurons was also observed, although NHPs exhibited no clinically apparent sensory deficits. There was no correlation between clinical findings and T-cell responses to the vector capsid or transgene product in NHPs. Piglets demonstrated no evidence of hepatic toxicity, but within 14 days of vector injection, all three animals exhibited proprioceptive deficits and ataxia, which profoundly impaired ambulation and necessitated euthanasia. These clinical findings correlated with more severe dorsal root ganglia sensory neuron lesions than those observed in NHPs. The liver and sensory neuron findings appear to be a direct consequence of AAV transduction independent of an immune response to the capsid or transgene product. The present results and those of another recent study utilizing a different AAV9 variant and transgene indicate that systemic and sensory neuron toxicity may be general properties of i.v. delivery of AAV vectors at high doses, irrespective of the capsid serotype or transgene. Preclinical and clinical studies involving high systemic doses of AAV vectors should include careful monitoring for similar toxicities.

Keywords: adeno-associated virus, gene therapy, hepatic toxicity, axonopathy, shock, liver failure

\section{INTRODUCTION}

MANY GENETIC AND ACQUIRED neuromuscular diseases involve degeneration of lower motor neurons. One of the most common and devastating examples is spinal muscular atrophy (SMA), an inherited deficiency of the survival of motor neuron (SMN) protein, characterized by progressive weakness resulting from selective death of lower motor neurons. The involvement of these cells in SMA and other disorders has made lower motor neurons critical targets for gene therapy. The cell bodies of lower motor neurons are distributed throughout the nuclei of cranial nerves and the ventral horn of the spinal cord, with axons projecting through peripheral nerves to reach neuromuscular junctions (NMJs). A variety of approaches have been evaluated to target these cells with gene therapy vectors. Intramuscular injection of adeno-associated virus

\footnotetext{
${ }^{*}$ Correspondence: Dr. James M. Wilson, Gene Therapy Program, 125 S. 31st Street, TRL 2000, Philadelphia, PA 19104-3403. E-mail: wilsonjm@upenn.edu
} 
(AAV) vectors has been demonstrated to transduce motor neurons at the NMJ, from which the vector undergoes retrograde transport to the neuron cell body. ${ }^{1-3}$ AAV-mediated transduction of motor neurons has also been achieved by injection into the cerebrospinal fluid, or by intravenous (i.v.) delivery of high doses of neurotropic AAV serotypes such as AAV9. ${ }^{4-11}$ AAV delivery i.v. may target motor neurons via peripheral axons, or by direct penetration into the central nervous system and transduction at the cell body. Systemic AAV9 administration has demonstrated successful motor neuron transduction in a variety of animal models, and results were recently reported from a first-inhuman trial of high-dose i.v. AAV9 in infants with SMA, in which patients have demonstrated remarkable improvements in survival and motor function compared to historical controls. ${ }^{11-14}$ In this trial, asymptomatic transaminase elevations (up to 35 times the upper limit of the normal range for alanine aminotransferase [ALT] and 37 times for aspartate aminotransferase [AST]) occurred in 4/15 patients, and were attributed to a T-cell response against capsid derived peptides presented by transduced hepatocytes. After this hepatic toxicity was observed, glucocorticoids were administered to subsequent subjects in order to attenuate T-cell responses to the vector capsid, similar to protocols employed in other clinical trials of systemic AAV gene therapy. ${ }^{15,16}$

AAV delivery i.v. was evaluated in juvenile nonhuman primates (NHPs) and piglets in order to understand better the efficiency and safety of this approach for targeting motor neurons in large animals. A neurotropic AAV vector closely related to AAV9 (AAVhu68) carrying a human SMN expression cassette was administered at a high dose to three juvenile NHPs and three piglets. Animals were evaluated for transduction of spinal motor neurons as well as evidence of toxicity.

\section{MATERIALS AND METHODS}

\section{Animal procedures}

All animal procedures were approved by the Institutional Animal Care and Use Committee of the University of Pennsylvania. Juvenile NHPs were group housed and provided additional enrichment. Piglets were also group housed, when compatible, and provided supplemental milk for appropriate weight gain. Animal welfare checks were performed by animal care staff twice daily. For i.v. administration, vector was infused via the saphenous vein (NHPs) or an ear vein (piglets) over 10 min. The day of vector infusion was designated study day 0 . Vectors were formulated in phosphatebuffered saline (PBS) with $0.001 \%$ Pluronic F68. On the day of injection, vectors were diluted in PBS (Corning) to deliver a dose of $2 \times 10^{14}$ genome copies $(\mathrm{GC}) / \mathrm{kg}$ in a volume $<5 \mathrm{~mL} / \mathrm{kg}$ (range $3.8-4.6 \mathrm{~mL} /$ kg; see Supplementary Tables S1 and S8 for details; Supplementary Data are available online at www.liebertpub.com/hum). Euthanasia was performed with i.v. pentobarbital overdose.

\section{Histology}

Tissues were fixed in formalin, paraffin embedded, sectioned, and stained with hematoxylin and eosin according to standard protocols. Tissues were evaluated histologically by a board-certified veterinary anatomic pathologist. In situ hybridization (ISH) was performed on formalin-fixed paraffinembedded tissues not exceeding a fixation time of $24 \mathrm{~h}$ using the ViewRNA ISH Tissue Assay Kit (Thermo Fisher Scientific) according to the manufacturer's protocol. Probes consisting of Z-shaped probe pairs were synthesized by the kit manufacturer. Probes specific for codon-optimized human $S M N$ were used either alone or in combination with probes for rhesus or pig ChAT as markers for motor neurons. Bound $S M N$ probes were detected by the formation of Fast Red precipitates imaged with a rhodamine filter set. ChAT probes were detected via Fast Blue deposits imaged with a custom filter set (AVR Optics) made according to the specifications of the kit manufacturer. Sections were counterstained with DAPI to show nuclei.

For immunohistochemistry, paraffin sections were deparaffinized through a series of xylene and ethanol, boiled in a microwave for $6 \mathrm{~min}$ in $10 \mathrm{mM}$ of citrate buffer ( $\mathrm{pH}$ 6.0), treated sequentially with $2 \%$ $\mathrm{H}_{2} \mathrm{O}_{2}$ (15 min; Sigma-Aldrich), avidin/biotin blocking reagents ( $15 \mathrm{~min}$ each; Vector Laboratories), and blocking buffer (1\% donkey serum in PBS $+0.2 \%$ Triton for $10 \mathrm{~min}$ ) followed by incubation with primary $(1 \mathrm{~h})$ and biotinylated secondary antibodies (45 min; donkey antibodies from Jackson ImmunoResearch) diluted in blocking buffer. Rabbit sera against fibrinogen (NBP1-33582; Novus Biologicals), CD3 (A045229-2; Agilent Technologies), or CD20 (PA5-16701; Life Technologies) served as primary antibodies. A Vectastain Elite ABC kit (Vector Laboratories) was used with 3,3'-diaminobenzidine (DAB) as substrate to visualize bound antibodies as brown precipitate. Sections were slightly counterstained with hematoxylin to show nuclei.

\section{Vector production}

AAVhu68 was isolated from a human source, and its nucleic acid sequence indicates it differs 
from that of AAV9 by two amino acids in the VP1/ VP2 N-terminus. The transgene cassette consisted of AAV2 inverted terminal repeats flanking a codonoptimized human $S M N$ cDNA expressed from a chicken beta-actin promoter with a cytomegalovirus immediate early enhancer and a chimeric intron, with a rabbit beta-globin polyadenylation sequence. Two AAVhu68 vector lots (PD174 and PD178) were used for this study. The vector was produced by triple transfection of adherent HEK293 cells using a previously described protocol. ${ }^{17}$ The vector was purified from supernatant by affinity chromatography using a POROS ${ }^{\mathrm{TM}}$ CaptureSelect ${ }^{\mathrm{TM}}$ AAV9 resin, followed by anion exchange chromatography to remove empty capsids and other impurities. Vectors were formulated in sterile PBS containing $0.001 \%$ pluronic F68 and stored at $\leq-60^{\circ} \mathrm{C}$. Genome copy titer measured by droplet digital polymerase chain reaction (PCR) was $4.56 \times 10^{13} \mathrm{GC} / \mathrm{mL}$ (PD178; triplicates were $4.44,4.52$, and $4.71 \times 10^{13} \mathrm{GC} / \mathrm{mL}$ ) and $6.06 \times 10^{13} \mathrm{GC} / \mathrm{mL}$ (PD174; triplicates were $5.56,5.48$, and $7.13 \times 10^{13} \mathrm{GC} / \mathrm{mL}$ ) for the two lots. Endotoxin was tested by LAL assay and was $<1 \mathrm{EU} /$ $\mathrm{mL}$ for both vector lots. Bioburden was evaluated by 5-day direct culture and was negative (no growth) for both vector lots. Viral capsid purity measured by sodium dodecyl sulfate polyacrylamide gel electrophoresis was $100 \%$ for both lots (Supplementary Fig. S1). Relative potency was determined by an in vitro transduction assay on Huh7 cells based on the formation of Gems and cytoplasmic structures. Copies of certificates of analysis for these two vector lots are provided in Supplementary Figs. S2 and S3. Analytical ultracentrifugation analysis of other preparations of this vector produced using the same process has shown that empty particles typically comprise $<10 \%$ of the total vector.

\section{Vector biodistribution}

At the time of necropsy, tissues were frozen on dry ice for vector biodistribution analysis. DNA was extracted, and vector genomes quantified by TaqMan PCR, as previously described. ${ }^{18}$

\section{Enzyme-Linked ImmunoSpot assay}

Interferon gamma Enzyme-Linked ImmunoSpot (ELISPOT) assay was performed, as previously described. ${ }^{19}$ The human SMN and AAVhu68 VP1 peptide libraries consisted of 15 mer peptides overlapping by five amino acids divided into two (SMN) or three (AAVhu68) pools.

\section{Neutralizing antibody assay}

Neutralizing antibodies against AAVhu68 were measured in serum, as previously described. ${ }^{20}$

\section{Cytokine analysis}

Cytokines were measured by Luminex assay using the 23-plex MILLIPLEX MAP Non-Human Primate Cytokine Magnetic Bead Panel according to the manufacturer's instructions.

\section{RESULTS \\ NHP study}

Three 14-month-old rhesus macaques were administered an i.v. dose of $2 \times 10^{14} \mathrm{GC} / \mathrm{kg}$ AAVhu68 vector expressing human $S M N$ under control of a chicken beta actin promoter with a cytomegalovirus immediate early enhancer (Supplementary Table S1). All animals exhibited stable vital signs during vector infusion and recovered uneventfully from anesthesia. At 6:42 a.m. on study day 4, animal $16 \mathrm{C} 176$ was reported by animal care staff as quiescent and minimally responsive while perched in its home cage; no abnormalities were noted when observed the previous afternoon. The animal was anesthetized at 7:25 a.m. for a physical exam, which revealed hypothermia, pale mucous membranes, an approximate $15 \%$ increase in body weight from the time of vector administration, and hepatomegaly. X-ray of the abdomen demonstrated free peritoneal fluid. Point of care bloodwork performed at 9:10 a.m. was notable for a packed cell volume of $22 \%$, glucose of $<20 \mathrm{mg} / \mathrm{dL}$, blood urea nitrogen (BUN) of $75 \mathrm{mg} /$ $\mathrm{dL}$, and creatinine of $2 \mathrm{mg} / \mathrm{dL}$. While under anesthesia, the animal was maintained on a warming blanket, and fluids were administered i.v. Despite supportive care, at 9:15 a.m., the animal exhibited signs of shock, including dyspnea and dilated pupils. The animal was euthanized at 9:25 a.m., and a complete necropsy was performed.

Blood was collected from the femoral vein following euthanasia, and a full serum chemistry panel performed (Supplementary Table S2). Remarkable findings included hypoproteinemia, markedly elevated liver enzymes (AST, ALT, gamma-glutamyltranspeptidase, alkaline phosphatase), hyperbilirubinemia, increased BUN, hyperphosphatemia, hypoglycemia, hypocalcemia, hypochloremia, hyperkalemia, hypocholesterolemia, hypertriglyceridemia, and elevated creatine phosphokinase (Fig. 1 and Supplementary Table S2). Severe hemolysis of the sample collected at necropsy limited interpretation of some chemistry results, such as potassium, and made it impossible to perform complete blood count (CBC) and coagulation studies. A multiplex cytokine panel revealed marked elevation of inflammatory cytokines (e.g., interleukin-6 increased from 0.2 to $6,490 \mathrm{pg} / \mathrm{mL}$ ) at the time of necropsy (Supplementary Table S3). 


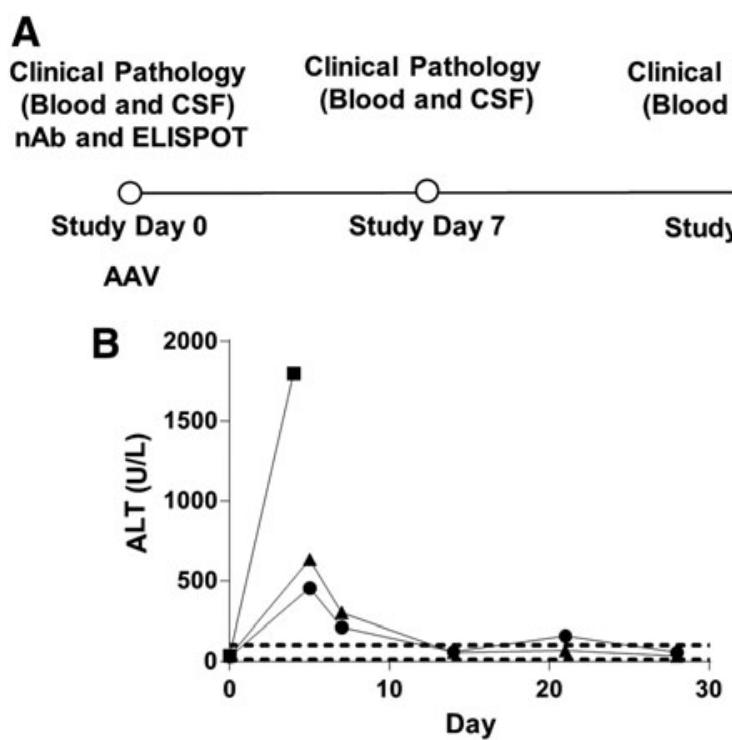

Clinical Pathology Clinical Pathology

(Blood and CSF) (Blood and CSF)
Clinical Pathology

(Blood and CSF) ELISPOT
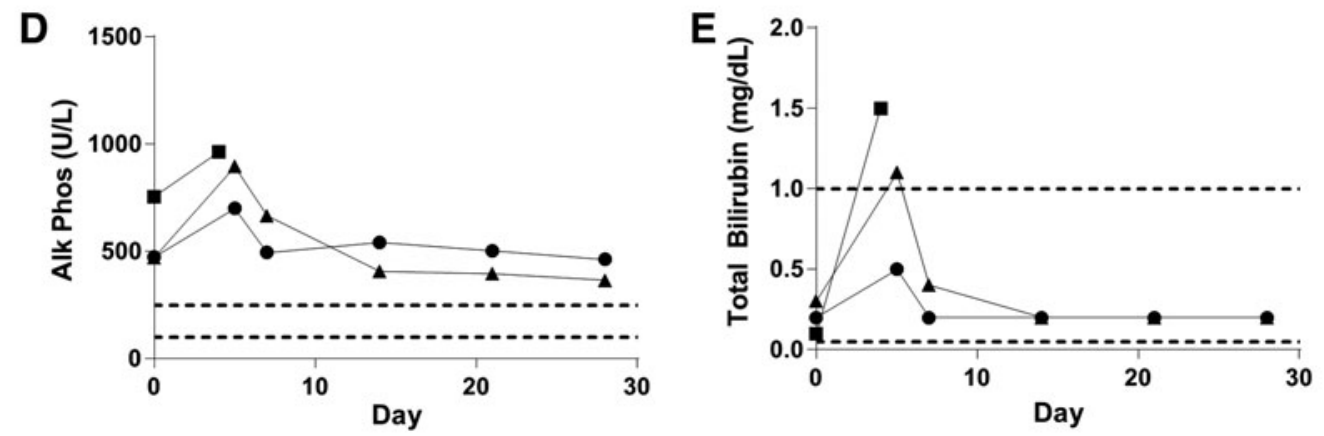

Figure 1. Acute transaminase elevations following intravenous (i.v.) administration of an adeno-associated virus (AAV) vector expressing human SMN to nonhuman primates (NHPs). (A) Study design, (B) serum alanine aminotransferase (ALT), (C) serum aspartate aminotransferase (AST), (D) serum alkaline phosphatase, and (E) serum total bilirubin. Unscheduled laboratory assessments were performed for all animals on study day 5 after animal $16 C 176$ developed acute liver failure requiring euthanasia. AST was not performed on study day 5 for animals $16 \mathrm{C} 116$ and 16C215. Dashed lines indicate laboratory reference range.

At necropsy, the abdominal cavity contained approximately $44 \mathrm{~mL}$ of red serosanguineous fluid. Cytologic evaluation of the abdominal fluid was consistent with transudate with acute hemorrhage. The liver was diffusely enlarged, firm, and mottled tan to red, which corresponded histologically to massive hepatocellular necrosis and degeneration affecting approximately $95 \%$ of the hepatic parenchyma, with only rare clusters of relatively normal hepatocytes remaining (Fig. 2A). The centrilobular to mid-zonal regions were severely congested with hepatocyte loss, degeneration, and necrosis. Additionally, there were small foci of fibrin, which occasionally formed plugs within sinusoids and filled the lumen of portal veins (acute fibrin thrombi; Fig. 2B and C). Immunohistochemistry (IHC) for fibrinogen confirmed the presence of sinusoidal fibrin plugs (Fig. 2D). Fibrinogen staining was strongly positive within periportal to mid-zonal regions, which were the areas of hepatocellular necrosis in the parenchyma that remained. Fibrinogen staining frequently highlighted sinusoids in these regions. The necrotic hepatocytes and associated debris were also strongly positive.

The spleen was diffusely enlarged, firm, and congested. The congestion was confirmed histologically. Germinal centers within the white pulp were depleted of lymphocytes with extensive cellular debris (lymphocytolysis) and had prominent, occasionally hyalinized, high endothelial venules. Similarly, the cortex of the thymus exhibited mild lymphocytolysis with prominent tingible body macrophages. Grossly, the mesenteric lymph nodes were diffusely enlarged and congested. Histologically, the follicles exhibited germinal center depletion with lymphocytolysis. Similar histologic findings were observed in other lymphoid tissues, including other lymph nodes (submandibular, rectal), thymus, bronchial-associated lymphoid tissue in the lung, and gut-associated lymphoid tissue in the colon and cecum. Lymph nodes associated with 

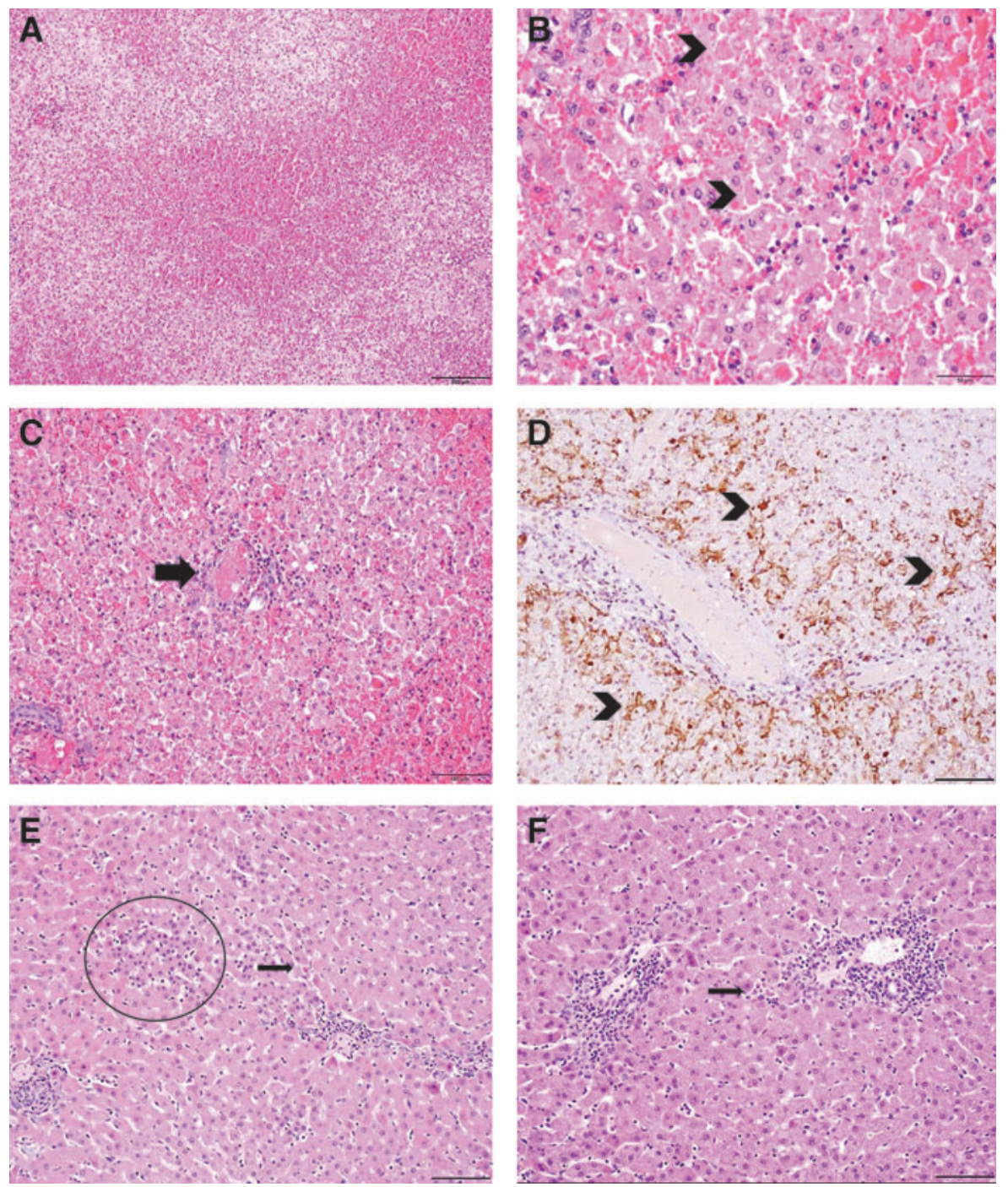

Figure 2. Liver histopathologic findings in juvenile NHPs treated with i.v. AAVhu68 expressing human SMN. Animal $16 \mathrm{C} 176$ required euthanasia on study day 4 and had massive acute hepatocellular necrosis (A) with sinusoidal fibrin deposition (B, arrowheads) and acute fibrin thrombi (C, arrow) in portal veins. Immunohistochemistry (IHC) for fibrinogen in animal 16C176 revealed prominent periportal sinusoidal fibrin deposition (D, arrowheads; fibrinogen IHC). The remaining two primates $(\mathrm{E}, 16 \mathrm{C} 215$; $\mathbf{F}$ 16C116) were clinically normal throughout the study, and both had similar findings in the liver consisting of single hepatocellular necrosis (arrows) predominantly surrounding portal areas with mild mononuclear cell infiltrates. Animal 16C215 (E) also had foci of hepatocellular regeneration (circle). Staining: hematoxylin and eosin; scale bar $=10 \mu \mathrm{m}$ (A and C), $50 \mu \mathrm{m}$ (B), and $100 \mu \mathrm{m}$ (D-F).

the rectum, assumed to be mesorectal lymph nodes, had subcapsular and medullary sinus erythrocytosis, consistent with drainage of red blood cells. The histologic findings within lymphoid follicles and thymic cortex were suggestive of severe systemic physiologic stress. Histologically, acute hemorrhage and edema was evident in the lung, adventitia of the gall bladder, heart, perirectal adipose tissue, and subcutis near the mammary gland. The superficial mucosa of the small intestines, cecum, and rectum were congested with occasional hemorrhage, as well as superficial aggregates of hemosiderin-laden macrophages. The presence of hemorrhage and edema in the lung, gastrointestinal tract, and liver were suggestive of shock, as well as a bleeding diathesis such as disseminated intravascular coagulation (DIC). ${ }^{21}$ Acinar cell degeneration in the pancreas was observed, which has also been reported in cases of shock in NHPs. ${ }^{21}$ Minimal adrenocortical singlecell degeneration and necrosis was present in the zona fasciculata of the adrenal glands, also likely attributable to systemic disease, such as systemic stress, ischemia, hemorrhage, and inflammation. ${ }^{22}$ No gross or histologic findings were noted in the brain, spinal cord, or cranial or peripheral nerves of this animal. No significant findings were observed in the kidneys.

The remaining two primates (16C116 and 16C215) were clinically normal throughout the study and were necropsied as planned on study day 28 . 
Bloodwork performed on study day 5 revealed ALT elevations of 454 and 632 IU/L for $16 \mathrm{C} 116$ and 16C215, respectively (reference range 10-100 IU/L). Serum ALT levels declined by study day 7 and normalized by study day 14 (Supplementary Table S2 and Fig. 1). Both animals demonstrated thrombocytopenia on study day 5 (16C116, 86,000/ $\mu \mathrm{L} ; 16 \mathrm{C} 215,24,000 / \mu \mathrm{L}$ ), although platelet clumping was noted on the peripheral blood smear for 16C116, potentially preventing accurate platelet quantification. Platelet counts normalized by study day 14 in both animals. Bloodwork was otherwise unremarkable throughout the study (Supplementary Table S2). A multiplex cytokine panel performed on study day 7 was unremarkable (Supplementary Table S3). At the time of necropsy, the liver of one animal (16C116) was mottled tan to red with an accentuated lobular pattern; no other significant gross abnormalities were observed in either of these two primates. Histologically, the liver of both primates exhibited minimal multifocal single hepatocellular necrosis, which was most prominent in the periportal region (Fig. $2 \mathrm{E}$ and $\mathrm{F}$ ). Additionally, animal 16C215 had multifocal aggregates of irregularly arranged hepatocytes with basophilic cytoplasm, vesicular nuclei, and occasional mitotic figures, indicative of regeneration (Fig. 2E). These hepatocyte clusters generally surrounded portal areas with interspersed mononuclear cell infiltrates and proliferating fibroblasts (fibroplasia).

Other pertinent histologic findings in the NHPs necropsied on study day 28 (16C116 and 16C215) were observed in the nervous system, predominately in the spinal cord, trigeminal nerve ganglia, and dorsal root ganglia (DRG) as well as the peripheral nerves, including the median, radial, sciatic, tibial, and peroneal nerves (Fig. 3 and Supplementary Tables S4-S6). The histologic lesions within the central and peripheral nervous system were often variable, with severity differing between tissue sections within the same segment, as well as between segments of spinal cord and peripheral nerves in the same animal. The DRG exhibited minimal to mild neuronal cell body degeneration characterized by dispersion of nissle substance and nucleus to the periphery (central chromatolysis), along with clustering of glial cells around neuronal cell bodies (satellitosis) and mononuclear cell infiltrates surrounding and infiltrating neuronal cell bodies (neuronophagia; Fig. 3B). Based on IHC, the mononuclear cell infiltrates were composed of CD3-positive T cells with few CD20positive B cells (Fig. 3C and D). Similar histologic findings were observed in the trigeminal nerve ganglia as well (not depicted). In both primates, the dorsal white matter tracts of the cervical, thoracic, and lumbar spinal cord exhibited bilateral, minimal to moderate axonopathy characterized by dilated myelin sheaths with and without myelomacrophages, indicating axonal degeneration (Fig. 3A). Occasionally, minimal to marked axonopathy was observed in the dorsal nerve roots of the spinal cord. The peripheral nerves (median, radial, sciatic, peroneal, and tibial) exhibited a similar axonopathy consisting of dilated myelin sheaths with and without myelomacrophages that ranged from mild to marked, along with variable mononuclear cell infiltrates and periaxonal fibrosis (Fig. 3E and F). Peripheral axonopathy was observed bilaterally. However, severity varied between sections and even within some nerve sections.

Analysis of vector biodistribution demonstrated gene transfer to all tissues evaluated, including the brain and spinal cord (Fig. 4). Notably, DRG were heavily targeted with $>1$ GC per diploid genome in all samples. Extremely high liver transduction was also evident, $>1,000 \mathrm{GC}$ per diploid genome. While AAV9 and its variants are viewed as neurotrophic, it is clear there is wide distribution when vector is delivered at high doses systemically. Vector DNA was detected at higher levels in the animal necropsied on study day 4 than those necropsied on study day 28, suggesting that some of the vector genomes detected in tissues early after injection do not represent stable transduction.

$S M N$ mRNA expression was evaluated by ISH (Fig. 5). There was marked $h S M N$ ISH staining in the liver of the animal necropsied on study day 4, but there was no detectable signal in the spinal cord or brain. Given the extremely high vector concentration in the liver of this animal, probe hybridization with vector DNA may have contributed to the ISH signal. The two animals necropsied on study day 28 exhibited robust $S M N$ expression in spinal motor neurons, with approximately 30 $90 \%$ of cells transduced on average at each level of the spinal cord evaluated. There were also scattered patches of transduced neurons and ependymal cells in the brain.

T-cell responses to the vector capsid and human SMN transgene product were evaluated by interferon gamma ELISPOT (Supplementary Table S7). Peripheral blood mononuclear cells (PBMCs) were collected for ELISPOT analysis prior to vector administration and at the time of necropsy for all three animals. Mononuclear cells were also harvested from the liver at necropsy. All samples exhibited a robust interferon gamma response to a positive control stimulation with PHA, with the exception of liver lymphocytes collected from animal 16C215, 

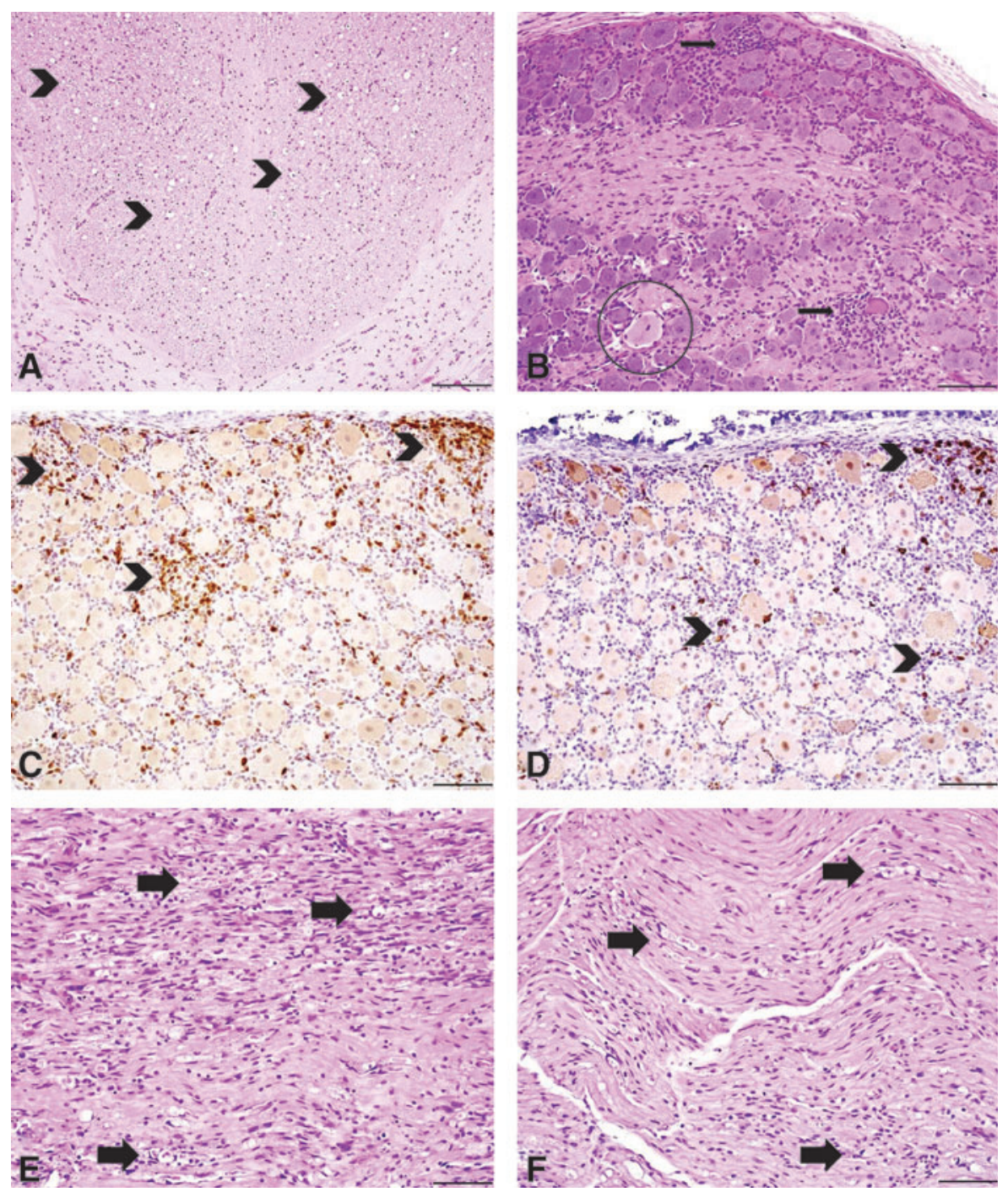

Figure 3. Representative central and peripheral nervous system histopathologic findings in juvenile NHPs treated with i.v. AAVhu68 expressing human SMN 28 days post injection, as depicted in images from animal 16C116. Both animals had an axonopathy (arrowheads) of the dorsal white matter tracts of the spinal cord (A). The dorsal axonopathy was typically bilateral and characterized by dilated myelin sheaths with and without myelomacrophages, consistent with axonal degeneration. The dorsal root ganglia (DRG) of the spinal cord (B) had minimal to mild neuronal cell body degeneration characterized by central chromatolysis (circle), satellitosis, and mononuclear cell infiltrates that surrounded and invaded neuronal cell bodies (neuronophagia; arrows). Mononuclear cell infiltrates were predominantly composed of CD3-positive T cells (C, arrowheads) with fewer CD20-positive B cells (D, arrowheads; CD3/CD20 IHC). A similar axonopathy (arrows) was observed in the peripheral nerves of the hind limb (sciatic nerve, E) and forelimb (median nerve, F). Animal $16 \mathrm{C} 176$ that was euthanized on study day 4 had no nervous system findings. Staining: hematoxylin and eosin; scale bar $=200 \mu \mathrm{m}$ (A), $100 \mu \mathrm{m}$ (B-F).

which exhibited no response to PHA stimulation and were excluded from subsequent analysis. No Tcell response to AAVhu68 capsid or hSMN was detectable at either time point for animals $16 \mathrm{C} 176$ or 16C215. Animal 16C116 exhibited no T-cell response in PBMCs at either time point, but had a detectable interferon gamma response to both the vector capsid and transgene product in lymphocytes harvested from the liver at the time of necropsy.

\section{Piglet study}

Three Micro-Yucatan piglets were administered an i.v. dose of $2 \times 10^{14} \mathrm{GC} / \mathrm{kg}$ AAVhu68 expressing human $S M N$ at 7 ( $n=1$; piglet A) or 30 ( $n=2$; piglets $\mathrm{B}$ and $\mathrm{C}$ ) days old, with piglet $\mathrm{A}$ being dosed before piglets B and C (Supplementary Table S8). All animals recovered uneventfully after vector administration. On study day 14 , piglet A developed hind-limb ataxia characterized by a swaying gait with intermittent knuckling and crossing over of the hind limbs. When the hind feet were placed on their dorsal surface, the piglet failed to correct positioning of the hoof (placement test), indicating a deficit in conscious proprioception. This ataxia progressed to the forelimbs over the course of $7 \mathrm{~h}$. This piglet also developed dyspnea, but ausculta- 


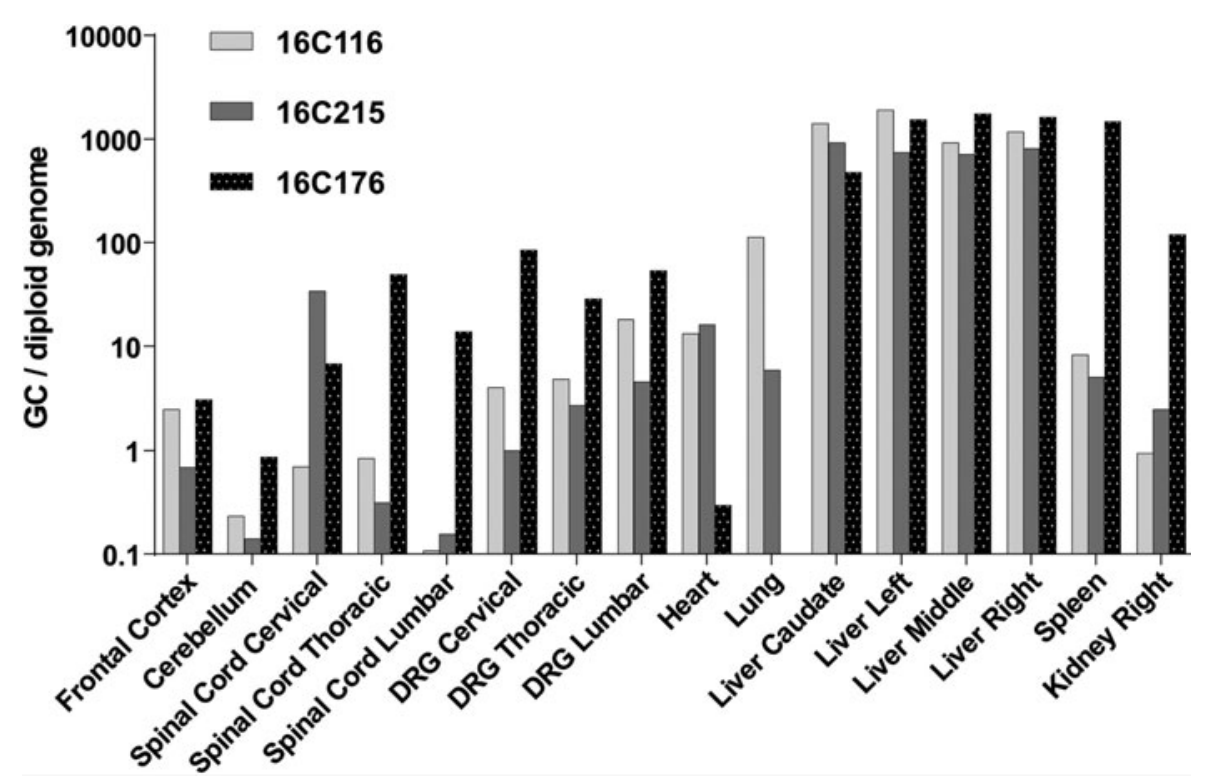

Figure 4. Vector biodistribution in rhesus macaques. Rhesus macaques treated with i.v. AAVhu68 expressing human $S M N$ were euthanized on study day 28 , except for animal 16C176, which was euthanized on study day 4. Vector genomes were detected in tissue DNA samples by quantitative polymerase chain reaction (PCR). Values are expressed as vector genome copies (GC) per host diploid genome. Data are shown for four liver lobes (caudate, left, middle, and right).
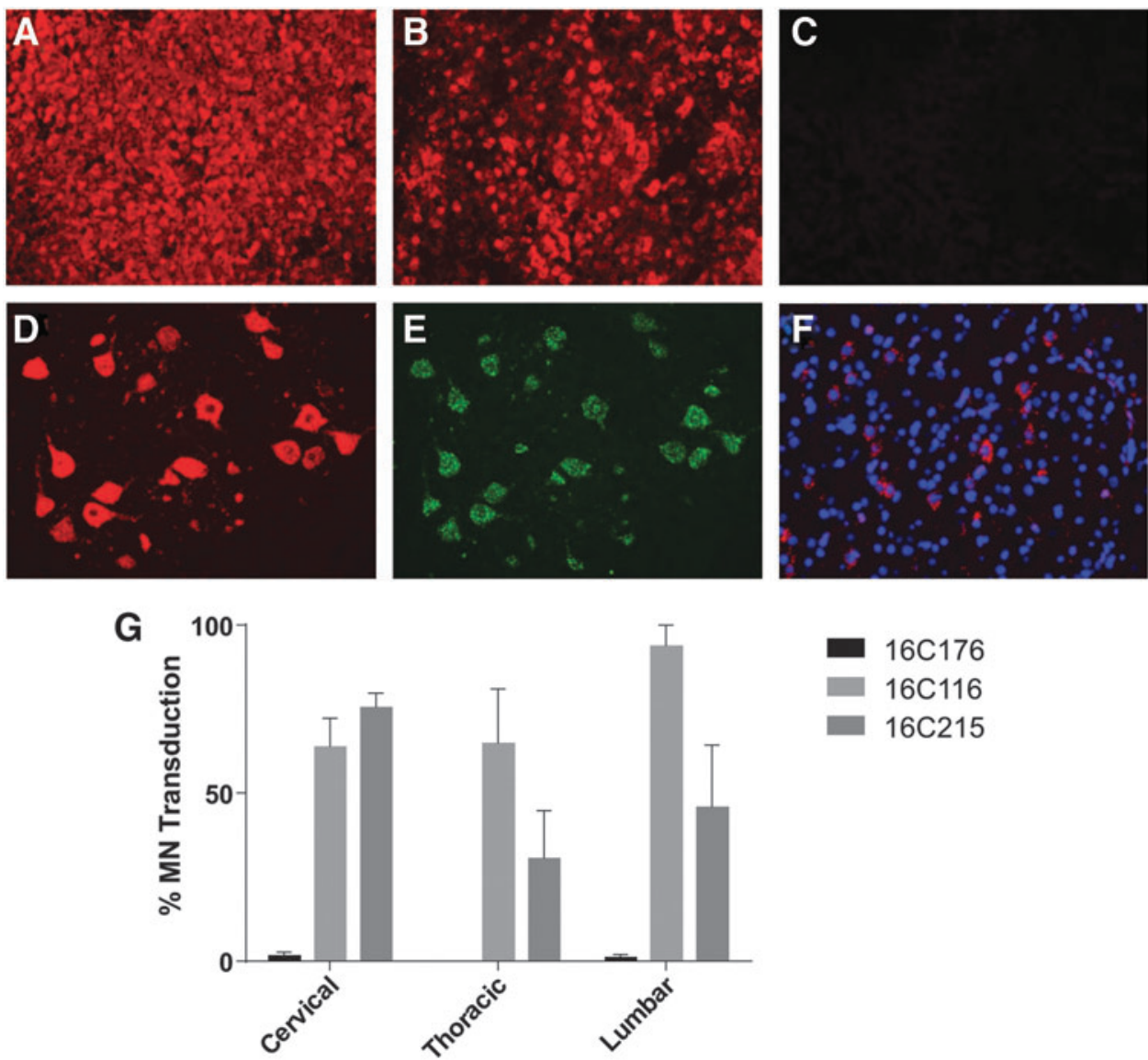

Figure 5. SMN expression in rhesus macaques. Human SMN RNA was detected by in situ hybridization (ISH) in the liver (A). The liver was stained with control probes for albumin (B) and green fluorescent protein (C). SMN-expressing cells were identified by ISH in the spinal cord (D). Motor neurons were identified by ChAT ISH (E). Rare patches of transduced neurons were detected by SMN ISH in the brain (F, DAPI nuclear stain). The percentage of ChAT+ motor neurons transduced at each level of spinal cord was quantified (G). Error bars=standard error of the mean. 
tion of all lung fields was within normal limits. The animal remained bright, alert, and responsive, but given the progression of neurological deficits, the piglet was euthanized on study day 14, and a full necropsy was performed.

On study days 11 and 12 , piglets $\mathrm{B}$ and $\mathrm{C}$, respectively, developed similar, albeit less severe, neurologic signs to those exhibited by piglet A. No dyspnea was observed in piglets B and C. Both piglets were euthanized on study day 13 due to progression of neurological signs, and a full necropsy was performed.

Piglet A was in fair to poor nutritional condition at the time of necropsy and had a clinical history of diarrhea on study day 7 , which resolved on study day 9 following treatment with intramuscular Ceftiofur. A CBC performed prior to euthanasia of piglet A revealed a marked neutrophilia $(22,468 /$ $\mu \mathrm{L}$; reference range $2,000-15,000 / \mu \mathrm{L})$. No other significant abnormalities were noted on bloodwork, including liver function tests (LFTs) and platelets (Supplementary Table S9). The neutrophilia likely corresponded to histologic evidence of bacterial bronchopneumonia. Bloodwork, including LFTs and platelets, was unremarkable for piglets B and C (Supplementary Table S9).

The cervical, thoracic, and lumbar DRG from all animals exhibited mild to marked neuronal cell body degeneration with mononuclear cell infiltrates (Fig. 6 and Supplementary Tables S4-S6). The histologic lesions of neuronal degeneration ranged from central chromatolysis with bright eosinophilic cytoplasmic globules to complete effacement with infiltrating mononuclear cells (neuronophagia) and satellitosis, as depicted in images from piglet B (Fig. 6B). Similar, albeit less severe, lesions were observed in the trigeminal nerve ganglia of all animals as well, ranging from minimal to moderate. In all piglets, the dorsal white matter tracts of the cervical, thoracic and lumbar spinal cord exhibited minimal to moderate axonopathy characterized by dilated myelin sheaths with and without myelomacrophages, consistent with axonal degeneration, as depicted in images from piglet $\mathrm{B}$ (Fig. 6A). The incidence of these lesions was the same across all animals. However, the severity was slightly decreased in one of the 30-day-old injected piglets (piglet C).
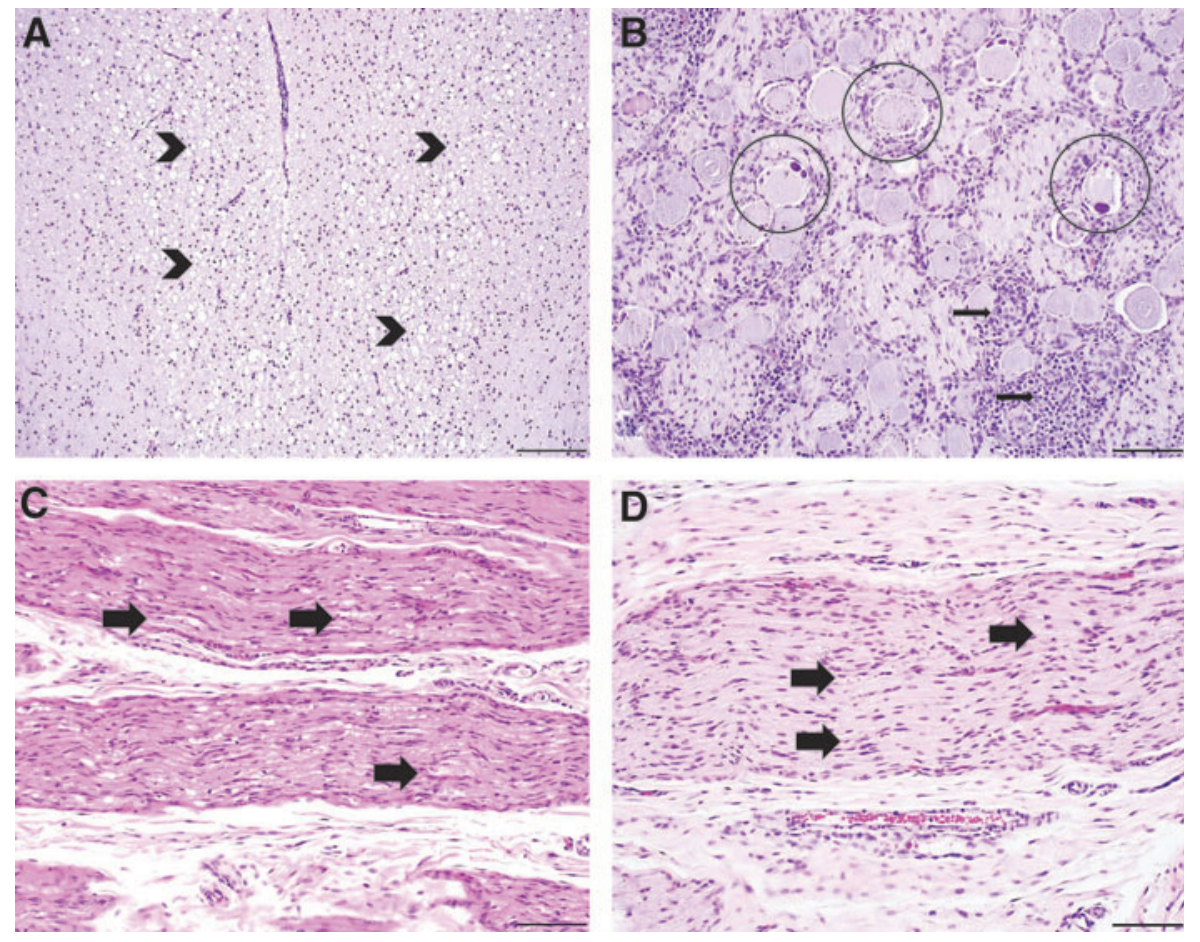

Figure 6. Representative histopathologic findings of piglets treated with i.v. AAVhu68 expressing human $S M N$ at 7 and 30 days of age. Piglets in both groups had an axonopathy of the dorsal white matter tracts of the spinal cord (A), as depicted in piglet B. The dorsal axonopathy was bilateral and characterized by dilated myelin sheaths with and without myelomacrophages, consistent with axonal degeneration (arrowheads). The DRG of the spinal cord (B) exhibited varying degrees of neuronal cell body degeneration characterized by central chromatolysis (circles), satellitosis, and mononuclear cell infiltrates that surrounded and invaded neuronal cell bodies (neuronophagia; arrows), as depicted in piglet B. A similar axonopathy (arrows) was observed to varying degrees in the peripheral nerves of the hind limb (sciatic nerve, C) and forelimb (median nerve, D) in the majority of piglets, as depicted in piglet A. Staining: hematoxylin and eosin; scale bar $=200 \mu \mathrm{m}$ (A), $100 \mu \mathrm{m}$ (B-D). 


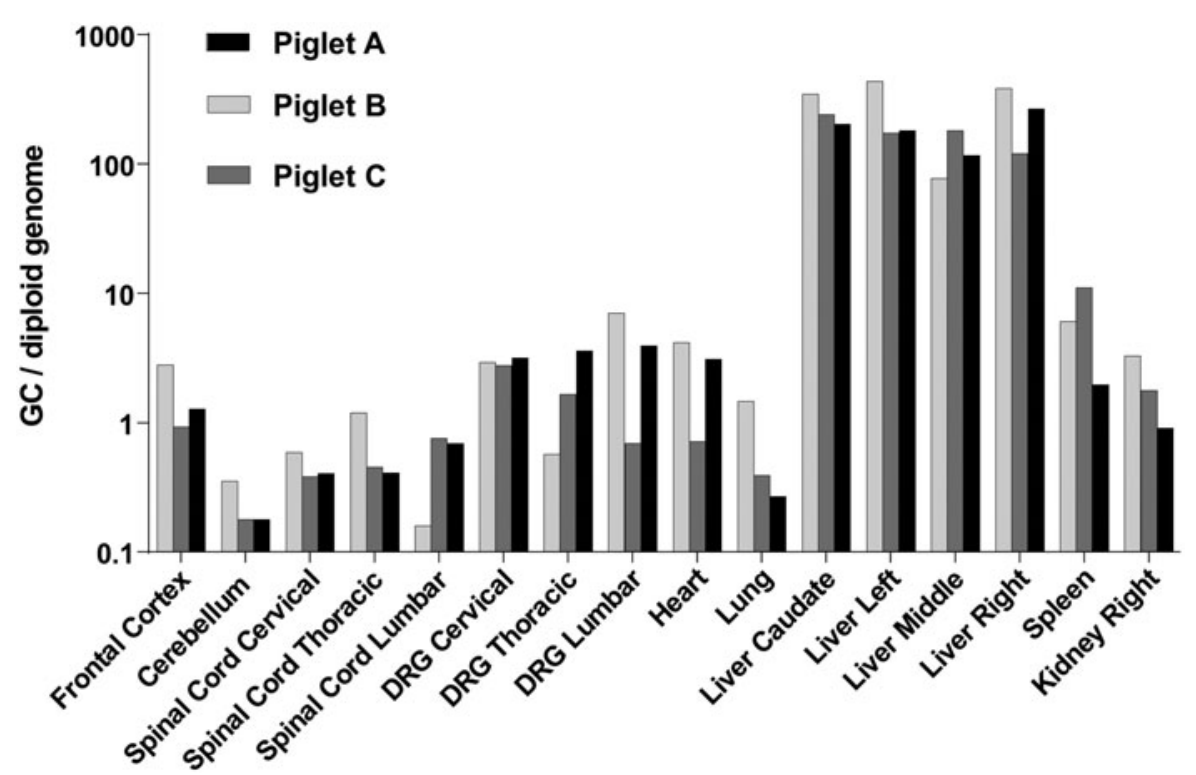

Figure 7. Vector biodistribution in piglets. Newborn piglets treated with i.v. AAVhu68 expressing human SMN were euthanized 13-14 days after injection. Vector genomes were detected in tissue DNA samples by quantitative PCR. Values are expressed as vector genome copies (GC) per host diploid genome. Data are shown for four liver lobes (caudate, left, middle, and right).

In piglet $\mathrm{A}$, the peripheral nerves (median, radial, sciatic, peroneal, and tibial) contained a similar axonopathy to the spinal cord that ranged from mild to severe bilaterally (Fig. 6C and D). Interestingly, the axonopathy in the peripheral nerves of the hind limbs (moderate to marked; Fig. 6C) was more severe than the forelimbs (mild; Fig. 6D). Piglet $\mathrm{B}$ exhibited a minimal peripheral axonopathy in most nerves examined, except for the peroneal nerve. The peripheral nerves of piglet $\mathrm{C}$ were histologically unremarkable. The histologic lesions within the central and peripheral nervous system were often variable, with severity differing between and within tissue sections of the same animal, as well as between different segments of spinal cord. No histologic abnormalities were noted in the brain or liver.

In general, the incidence and severity of the peripheral nerve lesions were decreased in piglets $\mathrm{B}$ and $\mathrm{C}$ compared to piglet $\mathrm{A}$, which coincided with less severe antemortem neurologic signs observed in the older animals. Additionally, severity of spi-
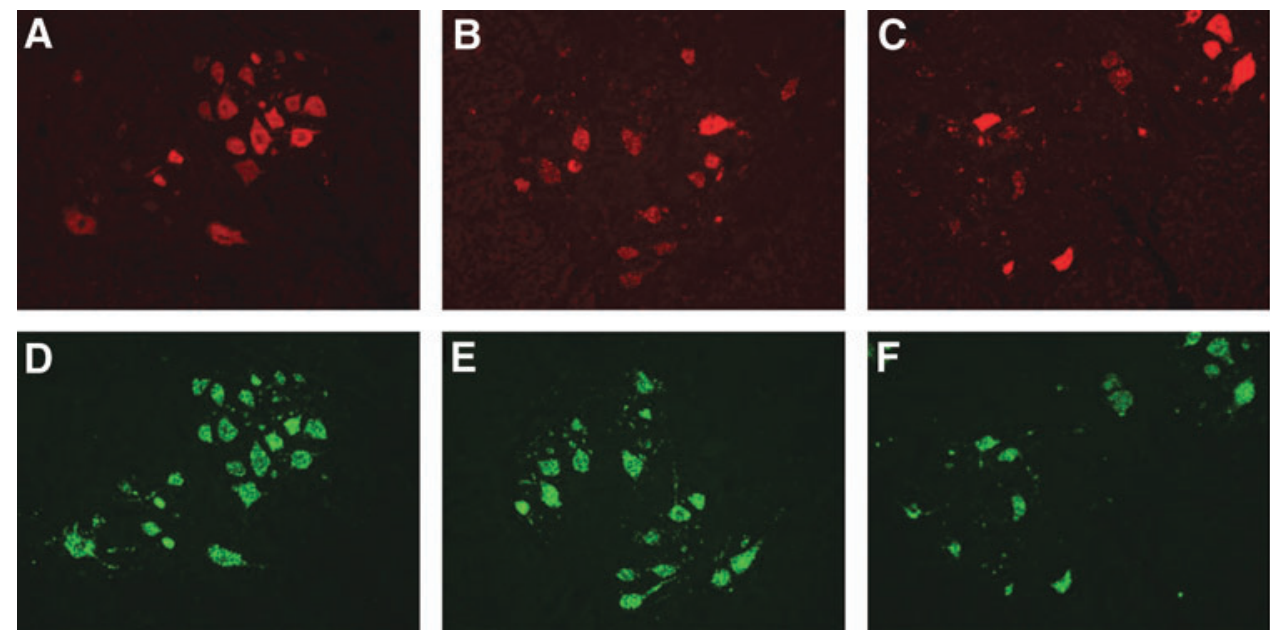

Figure 8. SMN expression in the spinal cord of piglets. Human SMN RNA was detected by ISH in cervical (A), thoracic (B), and lumbar (C) spinal cord segments. Motor neurons were identified by ChAT ISH in corresponding sections (D-F). Representative images are shown. 
nal cord lesions and incidence of peripheral nerve lesions were lowest in piglet $\mathrm{C}$, which also had the least severe neurologic signs.

Vector genomes were detectable in all tissues analyzed, including the brain and spinal cord (Fig. 7). Gene transfer to DRG was generally higher than that to spinal cord, often with $>1$ GC per host diploid genome. The highest vector genome copy numbers were found in the liver, with approximately 200 GC per diploid genome in liver samples from all three animals. There was no apparent impact of age at injection on the efficiency of gene transfer to the tissues evaluated.

Expression of human $S M N$ mRNA was detected by ISH in motor neurons throughout the spinal cord of all three animals (Fig. 8). The majority of motor neurons appeared to be transduced in all animals, with no apparent difference in transduction between the 7- and 30-day-old piglets.

\section{DISCUSSION}

Systemic delivery of AAV vectors has primarily focused on targeting hepatocytes, which are easily accessed via large fenestrations in the capillaries of the liver. Therapeutic levels of liver transduction have been achieved with AAV vector doses in the $10^{12}-10^{13} \mathrm{GC} / \mathrm{kg}$ range. ${ }^{15,16,23}$ Targeting organs without porous capillary networks, such as the skeletal muscle or the central nervous system, via intravascular routes requires quantities of vector that are 10- to 100-fold greater than those administered in most liver gene therapy trials (i.e., $\geq 10^{14} \mathrm{GC} /$ $\mathrm{kg}$ ). A variety of clinical studies are planned or have been initiated to evaluate the potential of high-dose i.v. administration of $\mathrm{AAV}$ vectors to transduce cells of skeletal muscle (NCT03199469, NCT03368742, and NCT03375164) and the central nervous system (NCT02122952 and NCT03315182).

The current study evaluated the efficiency and safety of AAVhu68, an AAV9 variant, expressing $S M N$ infused i.v. at a dose of $2 \times 10^{14} \mathrm{GC} / \mathrm{kg}$. Studies in adolescent NHPs and piglets showed potentially therapeutic levels of transduction in spinal motor neurons, although unexpected toxicities were encountered in each species. Acute systemic inflammation, coagulation defects, and hepatic toxicity were observed in NHPs, whereas sensory neuron degeneration occurred in both species with clinical sequelae evident only in piglets.

Two NHPs developed a rapid asymptomatic elevation of liver enzymes and bilirubin after vector administration, which spontaneously resolved by study day 14 . These animals also exhibited transient thrombocytopenia on study day 5 . One animal developed a more pronounced transaminase elevation in the context of systemic inflammation and shock requiring euthanasia on study day 4 . This animal exhibited evidence of DIC, with widespread hemorrhage, spontaneous hemoperitoneum, and hepatic fibrin deposition, although blood samples could not be obtained for confirmatory coagulation studies at the time of necropsy. At necropsy, the liver showed extensive necrosis, which could represent primary vector-related toxicity or which may be secondary to systemic inflammation or prolonged hypoperfusion in the setting of shock.

Recent studies in mature NHPs with AAVPHP.B, another AAV9 variant, revealed severe toxicity of potential relevance to this study. ${ }^{24}$ Administration i.v. of AAV9 and AAV-PHP.B vectors expressing green fluorescent protein was well tolerated at $2 \times 10^{13} \mathrm{GC} / \mathrm{kg}$. An animal treated with AAV9 at a dose of $7.5 \times 10^{13} \mathrm{GC} / \mathrm{kg}$ exhibited transient elevations in transaminases on study day 3 and at necropsy on study day $14^{24}$ that were asymptomatic without other toxicities. An animal administered i.v. AAV-PHP.B at a dose of $7.5 \times 10^{13}$ GC/kg acutely developed marked LFT abnormalities (ALT $>1,000 \mathrm{IU} / \mathrm{L}$ and bilirubin $>2.0 \mathrm{mg} / \mathrm{dL}$ ) associated with biochemical evidence of DIC and extensive hemorrhage requiring euthanasia on study day 5. Necropsy revealed diffuse bleeding into tissues and modest necrosis of the liver. It is unclear whether this animal would have ultimately developed shock, similar to $16 \mathrm{C} 176$, had it not been euthanized due to extensive bleeding.

The adverse events observed in these two NHP studies provide provocative but incomplete evidence for a unifying mechanism of toxicity resulting from high-dose systemic AAV administration. Findings common to both experiments include hepatocellular injury and development of a bleeding diathesis consistent with DIC within 5 days of vector administration. It is presently unclear whether liver damage is the primary insult leading to the coagulopathy, or if the coagulopathy is a manifestation of systemic toxicity that results in secondary liver damage.

Several mechanisms were considered by which AAV vectors could induce liver damage. The acute time course and ELISPOT results were inconsistent with activation of adaptive immunity and destruction of hepatocytes through antigen-specific T-cell responses to either the vector capsid or transgene product. Another possible mechanism is direct toxicity of the vector to hepatocytes, which could occur through activation of the DNA damage response by delivery of large numbers of vector genomes, or induction of an ER stress response by 
transgene overexpression. The findings in this study appear similar to those described in acute liver failure caused by acetaminophen toxicity, which is characterized by rapid hepatocellular degeneration and tissue factor presentation in the liver, which leads to intrahepatic coagulation. ${ }^{25-28}$ However, the systemic coagulopathy in the animal treated with high-dose AAV-PHP.B seems out of proportion to the extent of liver damage, suggesting that liver damage is not the only driver of the coagulopathy.

It is believed that AAV vectors are responsible for directly eliciting systemic inflammation leading to platelet activation and DIC independent of damage to the liver. Inflammatory cytokines were markedly elevated on study day 4 in the animal that developed acute liver failure and shock (16C176), although cytokine production may be secondary to shock and hepatic necrosis. Inflammatory cytokines were low to modestly elevated in the NHP treated with highdose AAV-PHP.B. However, steroid administration may have attenuated the inflammatory response. Previous studies have suggested that capsid and DNA components of an AAV vector are capable of activating innate immune pathways, although there has been little evidence to date to suggest that activation of these pathways could result in doselimiting toxicity in gene therapy applications. ${ }^{29,30}$ The timing and features of the toxicity noted in this NHP cohort are reminiscent of the innate immune response to high systemic doses of adenoviral vectors that resulted in lethal systemic inflammation in one research subject. ${ }^{31}$

The current hypothesis is that high systemic doses of AAV vectors lead to two independent insults to the host: direct damage to the hepatocyte and activation of systemic inflammation and an associated coagulopathy. It is possible that the vector activates cellular stress pathways that prime the hepatocyte for a more exaggerated response to inflammation. The critical question is the significance of these findings to human clinical trials of systemic AAV. The well-described transient elevations in transaminases associated with modest doses of systemic AAV in hemophilia clinical trials are not likely related to the findings, since these laboratory abnormalities occur much later (4-6 weeks) and are probably associated with adaptive immune responses. The two ongoing trials of high-dose systemic AAV for X-linked myotubular myopathy (MTM) and SMA both describe clinically asymptomatic transaminase elevations that have been treated with steroids. ${ }^{14,32}$ The timing of these abnormalities could have different prognostic implications. Early transaminase ele- vations (i.e., within the first week) may be a sign of systemic inflammation that could lead to the severe toxicity described in this report, while LFT abnormalities appearing later would be more consistent with adaptive immune responses observed in hemophilia trials that have remained without clinical consequences. In the SMA trial, transaminase elevations occurred within 1-4 weeks post vector administration without additional liver abnormalities or clinical manifestations ( $\mathrm{J}$. Mendell, pers. commun.). Another observation from the NHP study that is of relevance to clinical applications of highdose systemic AAV is the significant variation in toxicity observed between animals. The cause for this is unclear, although it is believed that it is more likely due to variation in the inflammatory response rather than differential susceptibility to hepatocellular injury, and could be related to previous immunological exposure to AAV or to genetic predisposition to an exaggerated inflammatory response. Finally, the impact of the age of the vector recipient on toxicity was not explored. Previous work with adenovirus vector suggested that newborn primates are less susceptible to systemic inflammation than older animals (unpublished observations).

The animal-to-animal variations observed in these studies and the rather steep toxicity curve in which only modest increases in vector dose led to qualitative changes in outcome create challenges in clinical trial design, especially when trials are expanded to larger patient populations. The fact that similar toxicities were observed in NHPs with two different AAV9 capsid variants carrying different transgenes suggests that this may represent a property of the AAV platform rather than idiosyncratic responses to specific vector constructs. It should not be surprising, however, that at high enough dose, one will see dose-limiting toxicities for any product. Unfortunately, it is difficult to compare vector doses used in different trials because of wide variations that exist in the PCR quantitation of vector used by different sponsors. This was illustrated by the characterization of a standard preparation of an AAV8 vector by a consortium of 16 laboratories that were asked to use standardized PCR protocols. ${ }^{33}$ The variation in titer of the reference standard among these laboratories was 100fold, indicating that it will be impossible to compare results of different trials based on vector dose. It is essential that key stakeholders in the field of gene therapy work together to develop standardized methods and reference standards. This will allow investigators in the field to leverage data sets better that emerge across different sponsors toward the development of safe and effective clinical trials. 
One potential but unlikely caveat to the findings is that impurities in the vector preparation, rather than the vector itself, may be the cause of toxicity. The vector release testing could exclude the presence of significant quantities of foreign proteins, endotoxin, or microbial contamination. However, other impurities could be present, and this study did not include direct experimental controls to account for toxicity related to impurities, such as animals treated with equivalent column fractions prepared from cell supernatant containing no vector. Notably, similar toxicity was observed in another study using vectors purified by iodixanol gradient centrifugation rather than the affinity chromatography method employed in the present study, indicating that contaminants specific to the purification process are not responsible for toxicity. ${ }^{17,24}$ Empty AAV capsids containing no vector genome represent another contaminant commonly found in AAV preparations. ${ }^{17}$ Empty capsids are not detected by PCR-based titering methods, and can thus lead to delivery of much larger quantities of viral protein than anticipated, potentially contributing to toxicity. The quantity of empty capsids in the vector lots utilized in this study was not evaluated, although several vector lots produced using the same method were shown to contain $<10 \%$ empty capsids by analytical ultracentrifugation. The previous observation of similar toxicity using vectors purified by iodixanol gradient centrifugation, which effectively eliminates empty capsids, further suggests that empty capsids are not the cause of the observed toxicity. ${ }^{17,24}$

To the authors' knowledge, DRG degeneration following AAV administration has not been studied or reported in published work, although a strong tropism for DRG has been noted in some studies. ${ }^{34,35}$ The lesions observed in the present study consisted of degeneration of DRG sensory neuron cell bodies as well as their central and peripheral axons. These lesions likely appear $>4$ days after gene transfer, as they were absent from the NHP sacrificed on study day 4 , and clinical signs first presented in piglets 11 days after vector administration. DRG lesions were slightly more severe in piglets than NHPs, whereas the degeneration of sensory axons in the spinal cord and peripheral nerves was similar to or even milder in the pigs compared to the NHPs, an observation that could be explained by the earlier necropsy time point in the piglets, which may not have allowed for degeneration of all axons associated with the dying cells. This hypothesis is supported by the NHP studies where axonopathy was noted in animals necropsied on study day 28 but not the animal ne- cropsied on study day 4 . Sensory deficits were apparent in piglets but not NHPs, suggesting that the extent of the DRG lesions in NHPs fell below a threshold at which clinical signs appear. The study did not characterize the type of sensory fibers affected in either species. Proprioception appeared to be impacted based on the deficits noted in piglets; impact on other sensory fibers was not clear, and should be further characterized in subsequent studies. It is difficult to determine whether the DRG toxicity observed in this study will translate to humans. Sensory symptoms have not been reported in any of the ongoing AAV clinical trials, though subclinical lesions similar to those in NHPs may not be noted on routine evaluations.

Encouraging clinical results have been observed following high systemic doses of AAV vectors in selected motor neuron and neuromuscular diseases (NCT03199469). ${ }^{14}$ It is fortunate that the doses of vectors needed to achieve efficacy in these trials, to date, have not been associated with dose-limiting toxicities. However, the present studies illustrate how severe and variable these toxicities can be with some AAV products in large animals. Additional work will be required to understand the mechanism(s) of the systemic and sensory neuron toxicities observed in these animal studies, and the relevance of each to human trials. At present, it is suggested that NHPs are the species of choice for evaluating systemic toxicity associated with highdose systemic AAV administration. An example of the use of NHPs in informing the design of a clinical trial is the systemic delivery of AAV8 expressing MTM1 in patients with MTM. Studies performed in infant NHPs dosed with the AAV8-MTM1 clinical vector at $8 \times 10^{14} \mathrm{GC} / \mathrm{kg}$ were relatively clean with no LFT abnormalities or coagulation defects, suggesting that the maximally tolerated dose was $>8 \times 10^{14} \mathrm{GC} / \mathrm{kg}$. The MTM clinical trial demonstrated encouraging results at the starting dose of $1 \times 10^{14} \mathrm{GC} / \mathrm{kg}$, which was at least eightfold lower than the maximally tolerated dose demonstrated in infant NHPs. ${ }^{32}$ Going forward, it is suggested that clinical trials of high-dose systemic AAV include careful preclinical vetting in NHPs and early laboratory and clinical evaluations for systemic toxicity, liver damage, and coagulopathy, as well as delayed symptoms of sensory neuropathy.

\section{ACKNOWLEDGMENTS}

We would like to thank Hongwei Yu, Mohamad Nayal, Yanqing Zhu, Erin Bote, and Amber Hamilton for technical assistance. We acknowledge the support of the Vector Core, Morphology Core, Immunology Core, and Program for Comparative 
Medicine at the University of Pennsylvania Gene Therapy Program, and the Penn Human Immunology Core (P30-CA016520).

\section{AUTHOR DISCLOSURE}

J.M.W. is an advisor to, a founder of, holds equity in, and has a sponsored research agreement with
REGENXBIO. He is a former consultant to and holds stock in Solid Biosciences. He also has a sponsored research agreement with Ultragenyx. In addition, he is a consultant to several biopharmaceutical companies. He is also an inventor on patents licensed to various biopharmaceutical companies. The remaining authors declare no potential competing financial interests.

\section{REFERENCES}

1. Lu YY, Wang LJ, Muramatsu S, et al. Intramuscular injection of AAV-GDNF results in sustained expression of transgenic GDNF, and its delivery to spinal motoneurons by retrograde transport. Neurosci Res 2003;45:33-40.

2. Hollis li ER, Kadoya $K$, Hirsch $M$, et al. Efficient retrograde neuronal transduction utilizing selfcomplementary AAV1. Mol Ther 2008;16:296-301.

3. Kaspar BK, Lladó J, Sherkat N, et al. Retrograde viral delivery of IGF-1 prolongs survival in a mouse ALS model. Science 2003:301:839-842.

4. Borel F, Gernoux G, Cardozo B, et al. Therapeutic rAAVrh10 mediated SOD1 silencing in adult SOD1(G93A) mice and nonhuman primates. Hum Gene Ther 2016;27:19-31.

5. Gray SJ, Nagabhushan Kalburgi S, McCown TJ, et al. Global CNS gene delivery and evasion of anti-AAV-neutralizing antibodies by intrathecal AAV administration in non-human primates. Gene Ther 2013:20:450-459.

6. Gurda BL, De Guilhem De Lataillade A, Bell P, et al. Evaluation of AAV-mediated gene therapy for central nervous system disease in canine mucopolysaccharidosis VII. Mol Ther 2016;24 206-216.

7. Samaranch L, Salegio EA, San Sebastian W, et al Strong cortical and spinal cord transduction after AAV7 and AAV9 delivery into the cerebrospinal fluid of nonhuman primates. Hum Gene Ther 2013; 24:526-532.

8. Hinderer $\mathrm{C}$, Bell $\mathrm{P}$, Vite $\mathrm{CH}$, et al. Widespread gene transfer in the central nervous system of cynomolgus macaques following delivery of AAV9 into the cisterna magna. Mol Ther Methods Clin Dev 2014;1:14051.

9. Meyer K, Ferraiuolo L, Schmelzer L, et al. Improving single injection CSF delivery of AAV9mediated gene therapy for SMA: a dose-response study in mice and nonhuman primates. Mol Ther 2015;23:477-487.

10. Gray SJ, Matagne V, Bachaboina L, et al. Preclinical differences of intravascular AAV9 delivery to neurons and glia: a comparative study of adult mice and nonhuman primates. Mol Ther 2011;19: 1058-1069.

11. Bevan AK, Duque S, Foust KD, et al. Systemic gene delivery in large species for targeting spinal cord, brain, and peripheral tissues for pediatric disorders. Mol Ther 2011;19:1971-1980.
12. Dominguez $E$, Marais $T$, Chatauret $N$, et al. Intravenous scAAV9 delivery of a codon-optimized SMN1 sequence rescues SMA mice. Hum Mol Genet 2011;20:681-693.

13. Foust KD, Wang X, McGovern VL, et al. Rescue of the spinal muscular atrophy phenotype in a mouse model by early postnatal delivery of SMN. Nat Biotechnol 2010;28:271-274.

14. Mendell JR, Al-Zaidy S, Shell R, et al. Single-dose gene-replacement therapy for spinal muscula atrophy. New Engl J Med 2017;377:1713-1722.

15. Nathwani AC, Tuddenham EGD, Rangarajan S, et al. Adenovirus-associated virus vector-mediated gene transfer in hemophilia B. New Engl J Med 2011;365:2357-2365.

16. George LA, Sullivan SK, Giermasz A, et al. Hemophilia B gene therapy with a high-specific-activity factor IX variant. New Engl J Med 2017;377:2215-2227.

17. Lock M, Alvira M, Vandenberghe LH, et al. Rapid, simple, and versatile manufacturing of recombinant adeno-associated viral vectors at scale. Hum Gene Ther 2010;21:1259-1271.

18. Wang L, Calcedo R, Bell P, et al. Impact of preexisting immunity on gene transfer to nonhuman primate liver with adeno-associated virus 8 vectors. Hum Gene Ther 2011;22:1389-1401.

19. Brantly ML, Chulay JD, Wang L, et al. Sustained transgene expression despite T lymphocyte responses in a clinical trial of rAAV1-AAT gene therapy. Proc Natl Acad Sci U S A 2009;106:16363-16368.

20. Calcedo R, Vandenberghe $L H, G a o ~ G$, et al. Worldwide epidemiology of neutralizing antibodies to adenoassociated viruses. J Infect Dis 2009;199:381-390.

21. Khan NA, Vierboom MPM, van Holten-Neelen C, et al. Mitigation of septic shock in mice and rhesus monkeys by human chorionic gonadotrophin-related oligopeptides. Clin Exp Immunol 2010;160:466-478.

22. Rushing GD, Britt RC, Britt LD. Effects of hemorrhagic shock on adrenal response in a rat model. Ann Surg 2006;243:652-654; discussion 654-656.

23. Miesbach W, Meijer K, Coppens M, et al. Gene therapy with adeno-associated virus vector 5-human factor IX in adults with hemophilia B. Blood 2017 Dec 15 [Epub ahead of print]; DOl: 10.1182/blood-2017-09-804419.

24. Hordeaux J, Wang 0, Katz N, et al. The neurotropic properties of AAV-PHP.B are limited to C57BL/6J mice. Mol Ther, in press. DOI: 10.1016/ j.ymthe.2018.01.018
25. Ganey PE, Luyendyk JP, Newport SW, et al. Role of the coagulation system in acetaminophen-induced hepatotoxicity in mice. Hepatology 2007;46:1177-1186.

26. Kerr R. New insights into haemostasis in liver failure. Blood Coagul Fibrinolysis 2003;14:S43-45.

27. Payen $C$, Dachraoui A, Pulce $C$, et al. Prothrombin time prolongation in paracetamol poisoning: a relevant marker of hepatic failure? Hum Exp Toxicol 2003:22:617-621.

28. James LP, Wells E, Beard RH, et al. Predictors of outcome after acetaminophen poisoning in children and adolescents. J Pediatr 2002;140:522-526.

29. Zhu J, Huang X, Yang Y. The TLR9-MyD88 pathway is critical for adaptive immune responses to adeno-associated virus gene therapy vectors in mice. J Clin Invest 2009;119:2388-2398.

30. Faust SM, Bell P, Cutler BJ, et al. CpG-depleted adeno-associated virus vectors evade immune detection. J Clin Invest 2013;123:2994-3001.

31. Raper SE, Chirmule N, Lee FS, et al. Fatal systemic inflammatory response syndrome in a ornithine transcarbamylase deficient patient following adenovira gene transfer. Mol Genet Metab 2003;80:148-158.

32. Audentes Therapeutics, Inc. Audentes announces positive interim data from first dose cohort of ASPIR0, a Phase $1 / 2$ clinical trial of AT132 in patients with X-linked myotubular myopathy. 2018. http:// investors.audentestx.com/phoenix.zhtml?c=254280\& $\mathrm{p}=$ irol-newsArticle\&ID=2324833 (last accessed February 2, 2018).

33. Ayuso $E$, Blouin V, Lock $M$, et al. Manufacturing and characterization of a recombinant adenoassociated virus type 8 reference standard material. Hum Gene Ther 2014;25:977-987.

34. Gray SJ, Kalburgi SN, McCown TJ, et al. Global CNS gene delivery and evasion of anti-AAVneutralizing antibodies by intrathecal AAV administration in non-human primates. Gene Ther 2013;20:450-459

35. Foust KD, Poirier A, Pacak CA, et al. Neonatal intraperitoneal or intravenous injections of recombinant adeno-associated virus type 8 transduce dorsal root ganglia and lower motor neurons. Hum Gene Ther 2008;19:61-70.

Received for publication January 18, 2018; accepted after revision January 26, 2018.

Published online: January 29, 2018 